\title{
Recent Results of Semi-inclusive DIS Experiments at Jefferson Lab
}

\author{
Kalyan Allada*† \\ Massachusetts Institute of Technology \\ E-mail: kalyan@jlab.org
}

\begin{abstract}
Semi-inclusive deep inelastic scattering (SIDIS) is a powerful tool to explore the 3-d structure of nucleon in momentum space. Through a combination of polarized or unpolarized lepton beam and nucleon target one can study various transverse-momentum dependent parton distribution functions (TMDs) that appear in the SIDIS cross-section. TMDs provide a description of nucleon structure in terms of parton's transverse momentum and its transverse spin, which enables us to study the quark orbital angular momentum effects in the nucleon. Several SIDIS experiments were performed in three experimental halls at JLab with $6 \mathrm{GeV}$ electron beam using both polarized or upolarized beam and target combinations. The kinematic range was mainly focued on valence quark region. In this proceeding we will discuss some of the recent results from JLab $6 \mathrm{GeV}$ run
\end{abstract}

XXIII International Workshop on Deep-Inelastic Scattering

27 April - May 12015

Dallas, Texas

\footnotetext{
* Speaker.

$\dagger$ This work is supported in part through funds provided by the U.S. Department of Energy under Contract Number DE-FG02-94ER40818.
} 


\section{Introduction}

The study of three dimensional nucleon structure in momentum space is one of the major goals of experimental research at JLab. Transverse momentum dependent parton distribution functions (TMDs) will provide us fundamental information about the nucleon structure by imaging partonic structure, its dynamics, and spin-orbit couplings in three-momentum space. At leading twist there are eight TMD quark distributions [1]. The first three, unpolarized $\left(f_{1}\right)$, helicity $\left(g_{1}\right)$ and transversity $\left(h_{1}\right)$ distributions are integrated over the transverse momenta of quarks, while the other five have an explicit dependence on the transverse momentum of quarks $\left(k_{T}\right)$. Inclusive DIS experiments performed in the past few decades provided very precise data on the first two PDFs ( $f_{1}$ and $g_{1}$ ), while the data on the remaining six TMDs are just beginning to appear from semi-inclusive deep inelastic scattering (SIDIS) experiments at HERMES, COMPASS and JLab. Of the eight leading-twist TMDs, transversity $\left(h_{1}\right)$, Sivers $\left(f_{1 T}^{\perp}\right)$, pretzelosity $\left(h_{1 T}^{\perp}\right)$, and $g_{1 T}$ can be accessed using a tranversely polarized target, while $h_{1 L}^{\perp}$ can be accessed using a longitudinally polarized target. The Boer-Mulders $\left(h_{1}^{\perp}\right)$ ) function is sensitive to the unpolarized SIDIS cross-section. We will discuss some of the recent single and double-spin asymmetry (SSA/DSA) results, along with cross-sections results from JLab SIDIS experiments.

\section{Unpolarized SIDIS Cross Sections}

In semi-inclusive deep inelastic scattering, $e N \rightarrow e^{\prime} h X$, a hadron is detected in coincidence with the scattered electron. This process factorizes into a convolution of initial parton distribution function in the nucleon with hard lepton-quark scattering, followed by quark hadronization. Typically, in SIDIS process a leading meson $(\pi$ or $K$ ) is detected that carries a large fraction $(z)$ of the exchanged virtual photon's energy. The detected meson allows us to tag the flavor of the struck quark in the nucleon and provide information on the specific quark momentum and spin distributions. The transverse momentum $\left(k_{T}\right)$ dependence of the struck quark can be inferred from the transverse momentum $\left(p_{T}\right)$ dependence of the detected meson in the final state.

The E00-108 experiment in Hall C measured pion electroproduction from both hydrogen and deuterium targets spanning the nucleon resonance region [2]. The kinematic ranges for the measured SIDIS cross sections for pions were: $0.2<x<0.6,2<Q^{2}<4\left(\mathrm{GeV} / c^{2}\right), 0.3<z<1$ and $P_{T}^{2}<0.2(\mathrm{GeV} / c)$. This experiment studied the low-energy factorization of SIDIS cross-section using quark-parton model, the $d_{v} / u_{v}$ of valence quarks from charged pion yields and the $P_{T}$ dependence of the SIDIS cross sections.

The $d_{v} / u_{v}$ ratio of for valence quarks were constructed from charged-pion cross sections measured on proton and deuteron, by taking the ratio of cross section difference of $\pi^{+}$and $\pi^{-}$from proton to that of deuteron (Fig. 1). The experimentally extracted ratio compared to expected ratios from CTEQ parton distributions found to be slightly low, but within the uncertainties. However, the ratio are in agreement with previous extraction from WA-21/25 and EMC experiments. The $P_{T}$ dependence of the cross sections are shown in Fig. 1. They were further studied using a simple model assuming only valence quarks and Gaussian distribution for transverse momentum dependence in quark distribution and fragmentation functions. Using this model the experiment extracted the transverse momentum widths of quark $\left(k_{T}\right)$ and FFs $\left(p_{T}\right)$. Under such assumptions the trans- 
verse momentum width of $u$-quark ( $k_{T}^{2}$ for $u=0.07 \pm 0.03(\mathrm{GeV} / \mathrm{c})^{2}$ ) is found to be larger than that of $d$-quark ( $k_{T}^{2}$ for $\left.d=-0.01 \pm 0.05(\mathrm{GeV} / \mathrm{c})^{2}\right)$, which is small and consistent with zero within the uncertainties. The transverse momentum $\left(p_{T}\right)$ widths of the favored and unfavored FFs are similar to each other and both larger than the quark widths.
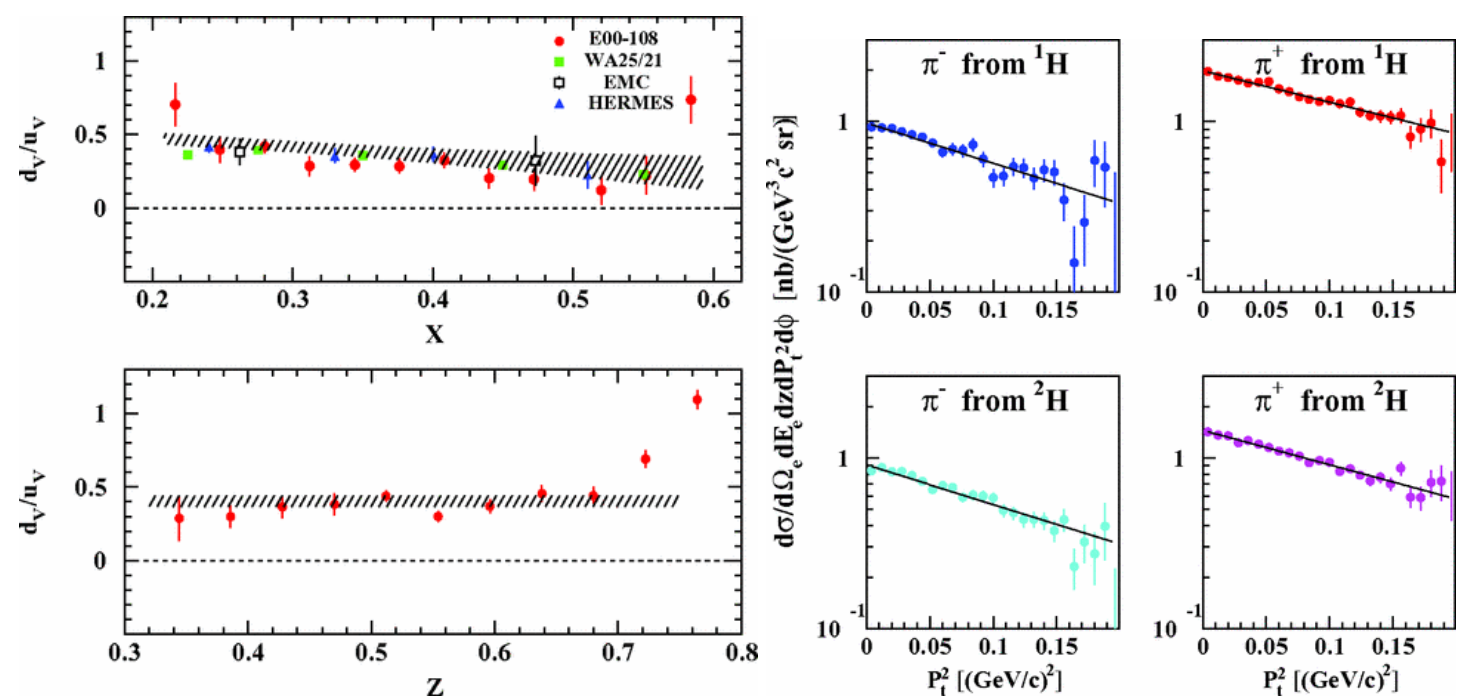

Figure 1: (Left-top) The ratio of valence quarks $d_{v} / u_{v}$ as a function of $x$ at $z=0.55$. Solid circles are data from the E00-108 experiment (at $\mathrm{P}_{T} \sim 0$ ), compared with other experiments. (Left-bottom) The ratio of valence quarks $d_{v} / u_{v}$ as a function of $z$ at $x=0.32$. (Right)The $P_{T}^{2}$ dependence of differential cross sections per nucleon for $\pi^{ \pm}$production on hydrogen and deuterium targets at $\langle z\rangle=0.55$ and $\langle x\rangle=0.32$. The solid lines are exponential fits. The error bars are statistical only [2].

\section{SIDIS Results Using Longitudinally Polarized Target}

Recent measurements of TMDs from longitudinally polarized target were made by CLAS collaboration at JLab. Some of the results include double-spin asymmetries and $A_{U L}^{\sin 2 \phi_{h}}$ moments of pions [5] in SIDIS, and $A_{L U}^{\sin \phi_{h}}$ moments of pions using longitudinally polarized beam and unpolarized proton target [6].

The $\sin 2 \phi_{h}$ moment of single-spin asymmetry involves a convolution of a leading twist TMD $h_{1 L}^{\perp}\left(x, k_{T}\right)$ and the Collins fragmentation funtion $H_{1}^{\perp}\left(z, p_{T}\right)$. It describes spin-orbit correlations of transversely polarized quarks in a longitudinally polarized nucleon. The SSA were measured using a polarized ammonia $\left({ }^{15} \mathrm{NH}_{3}\right)$ target and the CLAS detector in the kinematic range of $0.12<x<$ $0.48,0.9<Q^{2}<5.4\left(\mathrm{GeV} / c^{2}\right), 0.4<z<0.7$ and $P_{T}^{2}<0.112(\mathrm{GeV} / \mathrm{c})$. The extracted $\sin 2 \phi_{h}$ moment $A_{U L}^{\sin 2 \phi_{h}}$ is shown in Fig. 3 as a function of $x$ [5]. Non-zero moments are observed for $\pi^{+}$ channel while a relatively small amplitudes are observed for $\pi^{0}$. If Collins effect dominate in this process, then the SSA for $\pi^{-}$is expected to have opposite sign to that of $\pi^{+}$, but the data tends to have same sign as that of $\pi^{+}$, which seems to indicate that additional effects may contribute to this asymmetry.

The $\sin \phi_{h}$ moment $A_{L U}^{\sin \phi_{h}}$ is related to the twist-3 PDF $e(x)$ which is sensitive to quark-gluon correlations. The recent CLAS data of $A_{L U}^{\sin \phi_{h}}$ is shown in Fig. 3 [6]. The $\pi^{+}$and $\pi^{-}$data shows 
clear non-zero asymmetry with opposite sign, although, the $\pi^{-}$data has relatively small amplitude than that of $\pi^{+}$. The $\pi^{0}$ data has same sign as that of $\pi^{+}$, which is expected if one assumes $A_{L U}^{\sin \phi_{h}}$ for $\pi^{0}$ is the weighted average of $\pi^{+}$and $\pi^{-}$asymmetries . This data is also an improvement in precision over previous measurement from HERMES experiment [3].

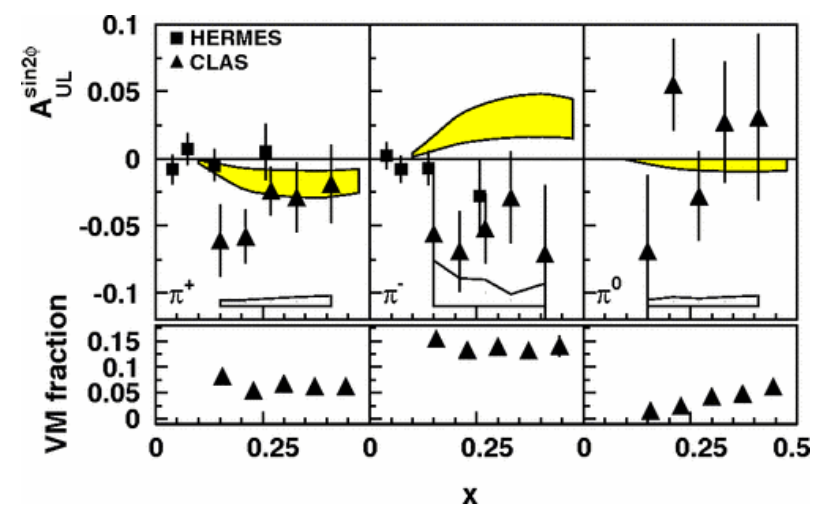

Figure 2: The longitudinal target SSA as a function of $x$ (triangles). The squares show the existing measurement of $\mathrm{A}_{U L}^{\sin 2 \phi_{h}}$ from HERMES. The lower band shows the systematic uncertainty. The upper band shows the existing theory predictions with uncertainties [5].

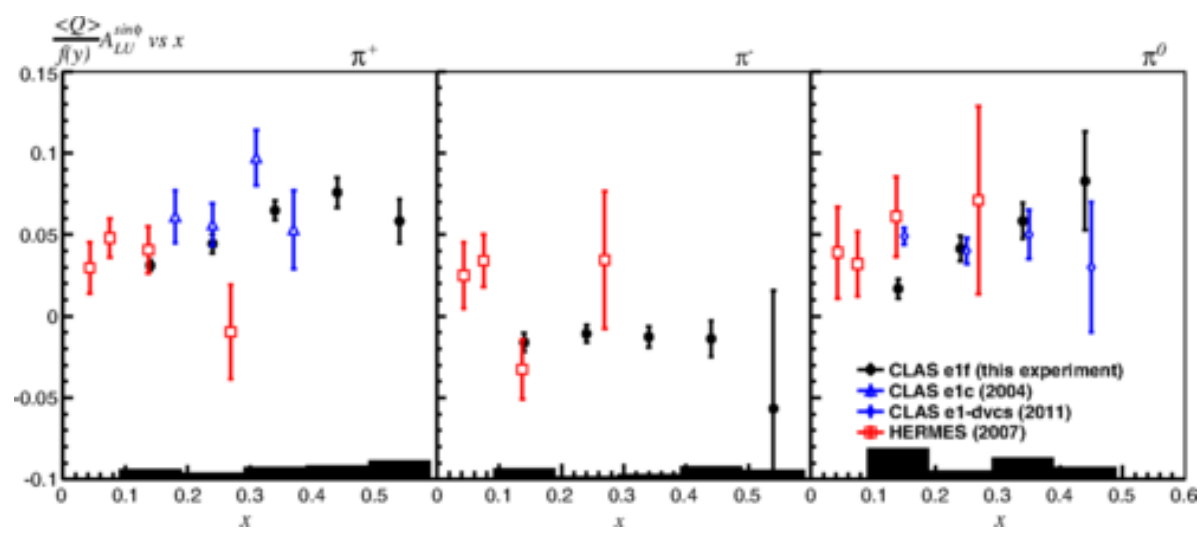

Figure 3: $A_{L U}^{\sin \phi_{h}}$ moments (solid black circles) are shown as a function of $x$ for charged and neutral pions [6]. The open (red) squares are from HERMES [3], and the open (blue) triangles are from previous CLAS publication [4].

\section{TMD Results from Transversely Polarized Target}

The first measurement of $A_{U T}$ (Collins, Sivers, pretzelosity) $[8,9,12]$ and $A_{L T}$ (worm-gear) moments [10] were performed by the E06-010 experiment in Hall A. The measurements were performed by scattering off longitudinally polarized electrons on a transversely polarized ${ }^{3} \mathrm{He}$ target in the SIDIS reaction ${ }^{3} \mathrm{He}\left(e, e^{\prime} h\right) X$, where $h$ is a charged hadron $\left(\pi^{ \pm}\right.$or $\left.K^{ \pm}\right)$. More recently, single and double spin asymmetries were also measured in the inclusive hadron channels $(e N \rightarrow h X)$ from the same experiment by making use of single-particle inclusive triggers [11,13]. We will discuss recent results of Collins and Sivers moments of kaons and the inclusive pion SSA and DSA in this section. 
E06-010 experiment used a $5.9 \mathrm{GeV}$ polarized electron beam and a polarized ${ }^{3} \mathrm{He}$ target to measure the SIDIS asymmetries. The beam helicity was flipped at $30 \mathrm{~Hz}$ while the target polarization direction was flipped every 20 minutes. For the SSA, unpolarized beam was achieved by summing together the two electron helicity states. Three sets of orthogonal Helmholtz coils were used to orient the target polarization direction in either horizontal or vertical direction in the plane transverse to the beam momentum.

The SIDIS event selection was done using PID and kinematic cuts. The following kinematical cuts were used: $Q^{2}>1.0(\mathrm{GeV} / \mathrm{c})^{2}, W>2.3 \mathrm{GeV} / \mathrm{c}^{2}$ and missing mass $W^{\prime}>1.6 \mathrm{GeV} / \mathrm{c}^{2}$. The neutron Sivers and Collins moments, and $\mathrm{A}_{L T}$ asymmetry were extracted from the ${ }^{3} \mathrm{He}$ results using the effective polarization method [14]. The method takes into account the initial-state nuclear effects. The proton dilution in the ${ }^{3} \mathrm{He}$ target was obtained by taking the ratio of yields for unpolarized hydrogen and ${ }^{3} \mathrm{He}$ targets in the SIDIS kinematics.

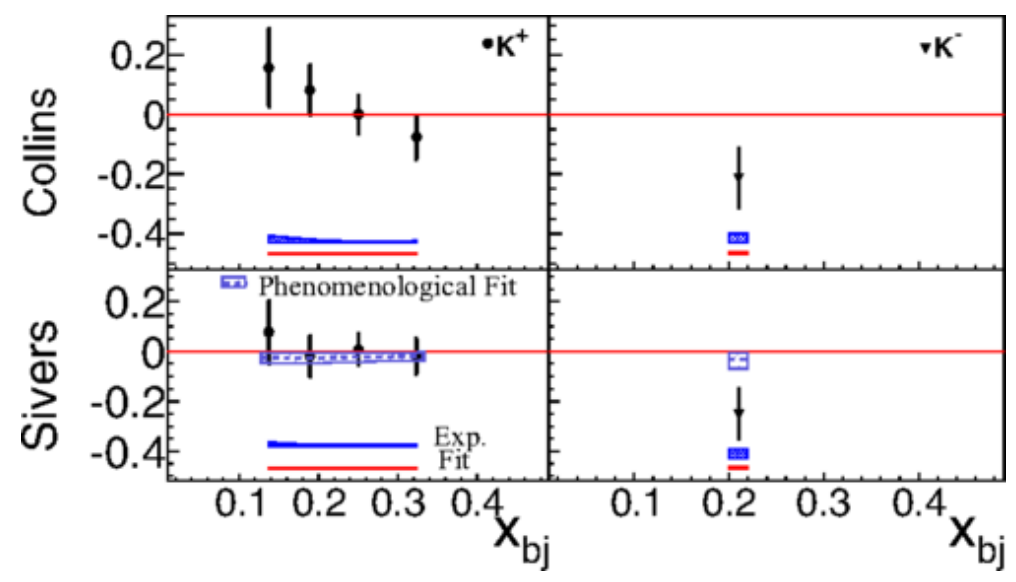

Figure 4: The extracted Collins and Sivers moments on ${ }^{3} \mathrm{He}$ are shown together with their statistical errors and systematic error bands for both $\mathrm{K}^{+}$and $\mathrm{K}^{-}$electro-production [9]. The Sivers moments are compared to theoretical predictions from a phenomenological fit to the world data.

The results for the extracted ${ }^{3} \mathrm{He}$ Collins and Sivers moments for kaons are shown in Fig. 4. The error bars represent statistical uncertainties. Experimental systematic uncertainties combined in quadrature from different sources are shown as a band labeled "Exp.". Systematic uncertainties due to neglecting other modulations are shown as a band labeled "Fit". The $K^{+}$Collins and Sivers moments are consistent with zero within error bars, while for $K^{-}$these moments are found to favor negative values at 2-sigma level. The Sivers moments from the ${ }^{3} \mathrm{He}$ target are compared to theoretical predictions from a phenomenological fit to the world data [15]. The $K^{+}$Sivers moments are consistent with the prediction, whereas $K^{-}$results differ from the prediction at the 2-sigma level. The uncertainities on kaon fragmentation functions are not fully estimated and the higher twist effects are not included in the the theoretical predictions. Due to the lack of information on the Collins fragmentation function for kaons, no theoretical predictions on the Collins moments are currently available. The data on the Collins moments will provide independent inputs for a future global analysis to extract flavor-dependent transversity distributions.

In addition to SIDIS, TMDs can also be studied using a less explored reaction of inclusive hadron production in lepton scattering, $l N \rightarrow h X$. Hall A E06-010 experiment collected data in this 
reaction and extracted $\mathrm{A}_{N}$ from SSA [11] and $\mathrm{A}_{L T}^{\cos \phi_{S}}$ from DSA [13]. $\mathrm{A}_{N}$ in $l N \rightarrow h X$ reaction has been studied assuming TMD factorization is valid, and found to be dominated by Sivers mechanism [17]. Non-zero $A_{N}$ was also estimated based on the twist-3 collinear factorization framework and in the SIDIS process by integrating over the scattered electron's azimuthal angle [16]. In the collinear factorization framework, $A_{L T}^{\cos \phi_{S}}$ in inclusive hadron production is an observable associated with twist- 3 effects. It can have twist-3 contributions from both the parton distributions inside the polarized nucleon and the parton fragmentation into final-state hadrons. By measuring $\mathrm{A}_{L T}^{\cos \phi_{S}}$, one can investigate the so-called worm-gear-type function $\tilde{g}(x)[18,19]$ as well as the role of quark-gluon-quark correlations in the nucleon and twist-3 effects in the fragmenting hadron.
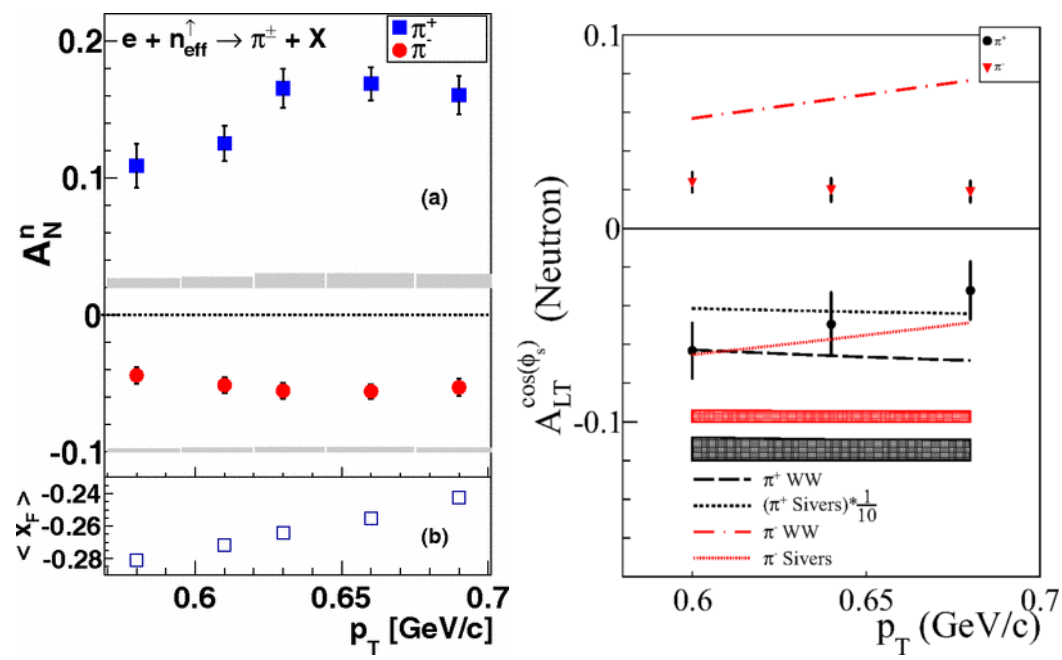

Figure 5: (Left): $A_{N}$ results on a neutron target extracted from the measured ${ }^{3} \mathrm{He}$ asymmetries. The solid band on the bottom of each panel shows the magnitude of the systematic uncertainty for each momentum bin. The lower plot (b) is the $\mathrm{x}_{F}$ and $\mathrm{p}_{T}$ correlation in this measurement [11]. (Right): Beam-target double spin asymmetries $\mathrm{A}_{L T}^{\cos \left(\phi_{s}\right)}$ for $\pi^{ \pm}$production from an effective neutron as a function of $\mathrm{p}_{T}$. The systematic uncertainty is shown as a band. The red (top, gray) band is the systematic uncertainty band for $\pi^{-}$, and the black (bottom) band is the systematic uncertainty band for $\pi^{+}$[13]. Predictions from collinear factorization by using two different scenarios [18] (Sivers function and Wandzura-Wilczek(WW)-type approximation) are shown as well.

The results for $\mathrm{A}_{N}$ for charged pions on an effective neutron target are shown on the left side of Fig. 5. The extracted $A_{N}$ is below $20 \%$ for both $\pi^{+}$and $\pi^{-}$, with the asymmetry amplitude for $\pi^{+}$ larger than those for $\pi^{-}$. Currently there are no theoretical predictions for low $p_{T}$ region (below 1 $\mathrm{GeV} / c$ ). The non-zero asymmetries for charged pions show a similar feature of flavor dependence to that observed for the Sivers asymmetry in SIDIS, and for $\mathrm{A}_{N}$ in $p p^{\uparrow} \rightarrow h X$ reaction.

The results of extracted neutron $\mathrm{A}_{L T}^{\cos \phi_{S}}$ for charged pions are shown on the right side of Fig. 5. The observed $\pi^{+}$and $\pi^{-}$asymmetries from effective neutron target are opposite in sign when the target polarization direction is in-plane transverse $\left(\phi_{S}=0^{\circ}\right)$. The asymmetry is expected to vanish when the traget spin is vertically polarized with respect to the scattering plane $\left(\phi_{S}=90^{\circ}\right)$. The theoretical calculations based on the two approximations shown in Fig. 5 give different predictions [18]. The data are consistent in sign with the prediction using the WW approximation, while the magnitude of the predictions is larger than that of the data. The calculation using the Sivers function is 
not consistent with the data. However, one needs to take into account the current uncertainty of the Sivers function and potential large next to leading order corrections, which are not included in the calculation.

\section{Summary}

We have discussed a few recent results of SSA and DSA from semi-incluisve DIS and inclusive hadron production expriements conducted at JLab. The upolarized cross-section data from Hall C has shown that at relatively low energies the parton information is still preserved in the SIDIS data. Non-zero $\sin 2 \phi_{h}$ from CLAS data indicate significant spin-orbit correlations of transversely polarized quarks in a longitudinally polarized proton. The non-zero $A_{L U}^{\sin \phi_{h}}$ indicate that the higher twist effects can be significant. The Hall A results for Collins and Sivers moments of negative kaons tend towards a negative asymmetry. The transvere SSA and DSA results from $e N \rightarrow \pi^{ \pm} X$ shows clear non-zero and large asymmetries. This process provides us an additional tool to study TMDs in lepton scattering. The JLab $12 \mathrm{GeV}$ upgrade will provide a unique opportunity to study all these TMDs with very high precision, extending the kinematics domain to higher $P_{T}$ and $Q^{2}$.

\section{References}

[1] A. Bacchetta. et al. JHEP 02093 (2007).

[2] R. Asaturyan. Phys. Rev. C85 (2012) 015202.

[3] A. Airapetian et al. (HERMES Collaboration), Phys. Lett. B 648, 164 (2007).

[4] H. Avakian et al. (CLAS Collaboration), Phys. Rev. D 69, (2004) 112004.

[5] H. Avakian et al. (CLAS Collaboration), Phys. Rev. Lett. 105 (2010) 262002.

[6] W. Gohn et al. (CLAS Collaboration), Phys. Rev. D. 89 (2014) 072011.

[7] M. Alekseev et al. Phys. Lett. B 692 (2010) 240.

[8] X. Qian. et al. Phys. Rev. Lett. 107 (2011) 072003.

[9] Y. Zhao. et al. Phys. Rev. C. 90 (2014) 055201.

[10] J. Huang. et al. Phys. Rev. Lett. 108 (2012) 052001.

[11] K. Allada. et al. Phys. Rev. C. 89 (2014), 042201(R).

[12] Yi. Zhang. et al. Phys. Rev. C. 90 (2014) 055209.

[13] Y. Zhao. et al. Phys. Rev. C. 92 (2015) 015207.

[14] S. Scopetta, Phys. Rev. D 75, (2007) 054005.

[15] M. Anselmino et al., Eur. Phys. J. A39, 89 (2009).

[16] B. Sun et al., Eur. Phys. J. C 65, 163 (2010).

[17] M. Anselmino et al., Phys. Rev. D 81, (2010) 034007.

[18] K. Kanazawa, A. Metz, D. Pitonyak, and M. Schlegel, Phys. Lett. B 742, 340 (2015).

[19] J. Zhou, F. Yuan, and Z.-T. Liang, Phys. Rev. D 81, 054008 (2010). 